\title{
Application of antigen cross-presentation research into patient care
}

\author{
Thijs W. H. Flinsenberg and Marianne Boes* \\ Laboratory of Translational Immunology, Department of Pediatrics, University Medical Center Utrecht, Utrecht, Netherlands \\ ${ }^{*}$ Correspondence: m.l.boes@umcutrecht.nl \\ Edited and reviewed by: \\ Christian Kurts, Friedrich Wilhelms-Universität Bonn, Germany
}

Keywords: immunotherapy, dendritic cell, cancer vaccines, clinical trial, virus infection, autoimmunity, T-cells

Dendritic cell (DC)-based cellular immunotherapy is being explored as a treatment modality for several malignancies, for viral diseases and auto-immune disorders. More than four decades of pre-clinical research on DC biology has cemented a strong foundation for clinical application of DC-based clinical trials, which already have been performed since the 1990s. Although sometimes met with limited patient success, clinical trials do yield better understanding of the requirements for optimal DC-based therapy. Recent advancements in the understanding of human DC biology and subset characteristics now give rise to ample opportunities to explore for a next generation of DC-based immunotherapy. This Research Topic is focused on articles that can help understanding the biology involved in DC antigen presentation, for future DC-based immunotherapy.

Dendritic cells are professional antigen presenting cells (APCs) that are particularly well endowed to elicit adaptive immune responses, via the presentation and cross-presentation of antigen-derived peptide/MHC complexes to T-lymphocytes. These processes decide how the host interacts with its environment, and therefore can be a target for pathogen interruption. van Montfoort et al. (1) provide an overview of cross-presentation features and describe how the study of various viral pathogens can elucidate anti-viral immune strategies. They further describe how DC maturation is crucial in immunity against viruses and how viruses may dampen this response to their own advantage. Understanding these presentation pathways is pivotal to develop effective DC-based immunotherapy.

Dendritic cell-based immunotherapy comes in two flavors. Either DCs are cultured and manipulated ex vivo before infusion, or endogenous DCs can be targeted in vivo. Concerning the latter approach, local administration of long peptides has proved effective in several diseases giving opportunity for further exploration. Both Delamarre and Cohn (2) and Rosendahl Huber et al. (3) discuss the requirements for improved antigen presentation, providing considerations on $\mathrm{CD} 4^{+}$and $\mathrm{CD} 8^{+}$activation, choice of antigen, and desired adjuvants. Delivering antigen to DCs is a next hurdle to take.

Regarding antigen delivery, it matters to engage responsive receptors, for these receptors can decide the intracellular pathway of antigen routing and enzymatic processing. Antigens are thereby directed toward assembly into peptide/MHC class I (crosspresentation) or peptide/MHC class II complexes, and induction of immunity or tolerance. Fehres et al. (4) describe the biology of receptor-mediated uptake in the context of antigen presentation, with special emphasis on C-type Lectin receptors. They further discuss the possibilities to formulate antigen in order to provide receptor-directed antigen delivery. Another import route of uptake involves the family of $\mathrm{Fc}$ receptors, which is discussed by Platzer et al. (5). Here, the role of this receptor family is highlighted in antigen presentation with emphasis on the opposing roles of activating and inhibiting Fc Receptor isoforms. Furthermore, they underscore that mechanisms of antigen presentation in mice are not always identical to the human pathways. Thus, the need for more research on human DC biology is warranted, for DC-vaccination strategies are still heavily based on mouse-biology.

When designing a DC-based immunotherapy, it is relevant to consider the subtype of DCs that one aims for. Boltjes and van Wijk (6) present an overview of all phagocyte subsets that are present throughout the human body in steady-state and under inflammatory conditions. They also emphasize the differences between mouse and human cells, and review cell types that should be considered for immunotherapy. Until recently, monocyte-derived DCs (MoDCs) were used mostly in DC-therapy, for their relative ease to culture in large quantities ex vivo. But while MoDCs can be found in human tissue under inflamed conditions, other DC subsets are more prevalent overall and may be more specifically endowed at stimulation of particular T-cell subsets, to be explored in immunotherapy. One subset that was suggested to be superior in $\mathrm{CD}^{+}{ }^{+}$T-cell priming is the recently identified BDCA-3 $3^{+}\left(\mathrm{CD} 141^{+}\right)$DC, characterized by CLEC9A and XCR1 expression. Tullett et al. (7) highlight recent findings explaining why these cells are effective at $\mathrm{CD} 8^{+} \mathrm{T}$-cell priming and discuss in vivo antigen targeting toward these DCs. Wimmers et al. (8) also describe the use of naturally circulating mDCs and pDCs for DC-based immunotherapy. They discuss the division of labor between $\mathrm{pDCs}$ and $\mathrm{mDC}$ and the clinical trials that are being performed using these subsets. Interestingly, they highlight that mDCs and pDCs work in synergy, supporting each other to enhance the effector phase of the adaptive immune response. Based on this observation, a next step in DC-based vaccination should include a cocktail of mDCs and pDCs, or in vivo antigen targeting to both subtypes.

Dendritic cells are often called "master regulators" of the immune response. Besides firing up immune reactions, DCs play an equally important role in the maintenance of peripheral tolerance, for example by dampening specific $\mathrm{T}$-cell responses or by inducing regulatory $\mathrm{T}$-cell subsets. Loss of tolerance is 


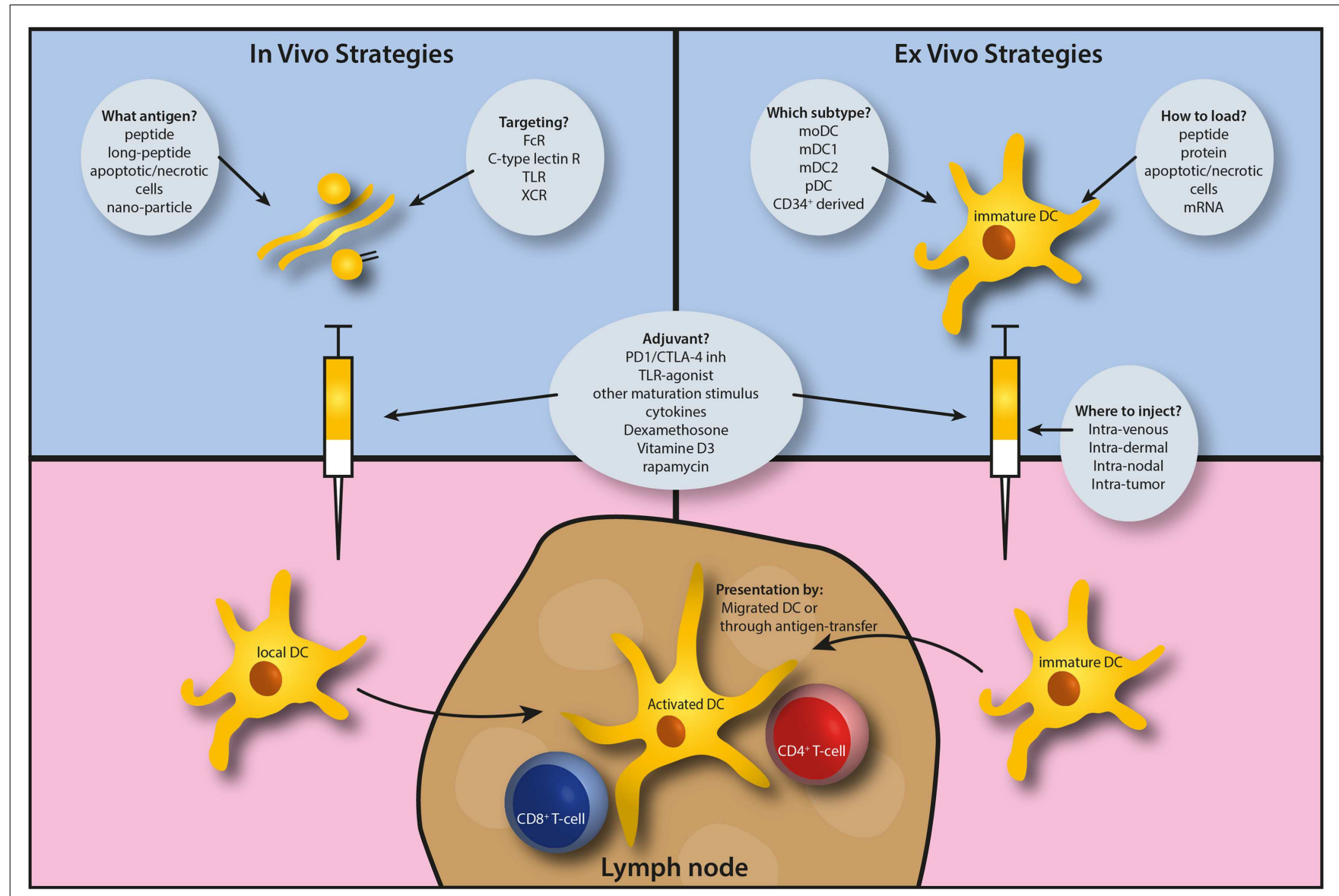

FIGURE 1 | Schematic outline of the considerations to apply antigen cross-presentation research to the clinic, most readily by dendritic cell-based immunotherapy.

of pivotal importance in auto-immune diseases as described by Hopp et al. (9). Their review concerns the presentation of self-antigen, which they discuss in the context of mechanisms in tolerance induction, DC maturation status, DC uptake and processing mechanisms, and tolerance-associated intracellular signaling pathways. The regulation of DC function is also controlled by metabolic pathways, as described by Everts and Pearce (10). Recent advancements concerning regulation of DC metabolism include the identification of key-proteins like PI3K, Akt, and mTOR in DC function. The awareness that manipulation of DC metabolic pathways changes DC function should be explored for designing DC-based cellular therapy, especially since it may give opportunity to steer toward more immunogenic or tolerogenic consequences. This could be of upmost importance in the setting of auto-immune diseases, anti-cancer, or graft-versus-host therapy.

Plantinga et al. (11) finally discuss recent developments in DCtherapy in the setting of allogeneic-hematopoietic cell transplantations (HCT). Such transplantations are considered a last-resort treatment for several malignancies of hematological origin. DCs grown from the same donor background as the HCT are now being explored for their potency to prevent cancer relapses early after allogeneic HCT. The various considerations for such DC vaccinations are discussed, such as the stem cell source, type of tumor antigen, and vaccination strategy.

The breadth and quality of the work discussed in this Research Topic underscores the strong translational push of DC research toward clinical settings (Figure 1). Immunotherapy is now being incorporated into standard cancer care, with antibody-based treatments currently being at more advanced stages than cellular therapies. The abundance of currently ongoing DC-based cellular immunotherapy trials should benefit patient care in the near future, as the roots for translational success are implanted in well-established pre-clinical research settings.

\section{REFERENCES}

1. van Montfoort N, van der Aa E, Monique Woltman A. Understanding MHC class I presentation of viral antigens by human dendritic cells as a basis for rational design of therapeutic vaccines. Front Immunol (2014) 5:182. doi:10.3389/fimmu.2014.00182

2. Delamarre L, Cohn L. Dendritic cell-targeted vaccines. Front Immunol (2014) 5:255. doi:10.3389/fimmu.2014.00255

3. Rosendahl Huber S, van Beek J, de Jonge J, Luytjes W, van Baarle D. T cell responses to viral infections - opportunities for peptide vaccination. Front Immunol (2014) 5:171. doi:10.3389/fimmu.2014.00171

4. Fehres M, Unger WWJ, Garcia-Vallejo JJ, van Kooyk Y. Understanding the biology of antigen cross-presentation for the design of vaccines against cancer cynthia. Front Immunol (2014) 5:149. doi:10.3389/fimmu.2014.00149 
5. Platzer B, Monique Stout M, Fiebiger E. Antigen cross-presentation of immune complexes. Front Immunol (2014) 5:140. doi:10.3389/fimmu.2014.00140

6. Boltjes A, van Wijk F. Human dendritic cell functional specialization in steadystate and inflammation. Front Immunol (2014) 5:131. doi:10.3389/fimmu.2014. 00131

7. Tullett K, Lahoud M, Radford KJ. Harnessing human cross-presenting CLEC9A+XCR1+ dendritic cells for immunotherapy. Front Immunol (2014) 5:239. doi:10.3389/fimmu.2014.00239

8. Wimmers F, Schreibelt G, Sköld AE, Figdor CG, de Vries IJM. Paradigm shift in dendritic cell-based immunotherapy: from in vitro generated monocytederived DCs to naturally circulating DC subsets. Front Immunol (2014) 5:165. doi:10.3389/fimmu.2014.00165

9. Hopp A-K, Rupp A, Lukacs-Kornek V. Self-antigen presentation by dendritic cells in autoimmunity. Front Immunol (2014) 5:55. doi:10.3389/fimmu.2014. 00055

10. Everts B, Pearce EJ. Metabolic control of dendritic cell activation and function: recent advances and clinical implications. Front Immunol (2014) 5:203. doi:10.3389/fimmu.2014.00203

11. Plantinga M, de Haar C, Nierkens S, Jan Boelens J. Dendritic cell therapy in an allogeneic-hematopoietic cell transplantation setting: an effective strategy toward better disease control? Front Immunol (2014) 5:218. doi:10.3389/fimmu. 2014.00218

Conflict of Interest Statement: The authors declare that the research was conducted in the absence of any commercial or financial relationships that could be construed as a potential conflict of interest.

Received: 26 May 2014; accepted: 03 June 2014; published online: 17 June 2014.

Citation: Flinsenberg TWH and Boes $M$ (2014) Application of antigen cross-presentation research into patient care. Front. Immunol. 5:287. doi: 10.3389/fimmu.2014.00287

This article was submitted to Antigen Presenting Cell Biology, a section of the journal Frontiers in Immunology.

Copyright $(2014$ Flinsenberg and Boes. This is an open-access article distributed under the terms of the Creative Commons Attribution License (CC BY). The use, distribution or reproduction in other forums is permitted, provided the original author(s) or licensor are credited and that the original publication in this journal is cited, in accordance with accepted academic practice. No use, distribution or reproduction is permitted which does not comply with these terms. 